\title{
Three-Component Suzuki-Knoevenagel Synthesis of Merocyanine Libraries and Correlation Analyses of Their Oxidation Potentials and Optical Band Gaps
}

\author{
Tim Meyer, Roxanne Krug and Thomas J. J. Müller *(D)
}

Citation: Meyer, T.; Krug, R.; Müller, T.J.J. Three-Component Suzuki-Knoevenagel Synthesis of Merocyanine Libraries and Correlation Analyses of Their Oxidation Potentials and Optical Band Gaps. Molecules 2021, 26, 5149 https: / / doi.org/10.3390/ molecules26175149

Academic Editors: Ana Maria

Gomez Neo and Carlos

Fernández Marcos

Received: 16 July 2021

Accepted: 19 August 2021

Published: 25 August 2021

Publisher's Note: MDPI stays neutral with regard to jurisdictional claims in published maps and institutional affiliations.

Copyright: (c) 2021 by the authors. Licensee MDPI, Basel, Switzerland. This article is an open access article distributed under the terms and conditions of the Creative Commons Attribution (CC BY) license (https:/ / creativecommons.org/licenses/by/ $4.0 /)$.
Institut für Organische Chemie und Makromolekulare Chemie, Heinrich-Heine-Universität Düsseldorf, Universitätsstrasse 1, D-40225 Düsseldorf, Germany; tim-meyer@outlook.com (T.M.); roxanne.krug@web.de (R.K.)

* Correspondence: ThomasJJ.Mueller@uni-duesseldorf.de; Tel.: +49-(0)211-81-12298

\begin{abstract}
The Suzuki coupling Knoevenagel condensation one-pot synthesis of boronic acids/esters, (hetero)aromatic bromo aldehydes and methylene active compounds is a highly practical consecutive three-component process to provide substance libraries with 60 donor- $\pi$-bridge-acceptor molecules, i.e., merocyanines in a broader sense, in moderate to excellent yield. As already seen with the naked eye, a broad variation of the optical properties becomes accessible using this practical synthetic tool. More systematically, correlation analyses upon plotting the optical band gaps against the first oxidation potentials of redox active systems of consanguineous series furnishes linear correlations and, by extension, two parameter plots (oxidation potential and emission maximum) planar correlations with the optical band gaps.
\end{abstract}

Keywords: absorption; aldol condensation; correlation analysis; cross-coupling reactions; cyclic voltammetry; fluorescence

\section{Introduction}

Multicomponent reactions (MCR) [1-4], conducted in domino, sequential or consecutive fashion, represent a powerful, efficient and efficacious tool for constructing complex molecular scaffolds in a one-pot fashion. In recent years, we have developed and explored MCRs as concise entries to functional chromophores. In particular, for fluorophores and electrophores [5-7], MCRs are most fruitfully applied in the sense of a chromogenic approach, which results directly in the formation of the functional chromophore of interest.

Among functional chromophores, merocyanines [8-13] are particularly interesting. In a very general sense, merocyanines can be structurally characterized as donor- $\pi$-bridgeacceptor scaffolds with high extinction coefficients, and as chromophores they are omnipresent in optoelectronics [14-17], organic semiconductors [18] and organic photovoltaics (OPV) [19]. Due to their inherent dipolar nature, the self-assembly of merocyanines in solution even allows for concise access to nanoscale objects and supramolecular materials [20,21]. The classical synthesis of merocyanines, as for many polymethine dyes, is governed by conventional aldol or Knoevenagel condensations [8,22-24].

Besides MCR syntheses of indolone [25-27], aroyl [28,29] and coumarin [30]-based merocyanines, we just recently communicated the versatile access to DSSC (dye sensitized solar cell) merocyanines bearing carboxylic acid end groups for immobilization on $\mathrm{TiO}_{2}$ by a consecutive three-component Suzuki-Knoevenagel condensation sequence [31].

Since merocyanines are outstandingly important in OPV [19], the effect of acceptor strength in molecular architectures on the morphology of bulk-heterojunction fullerene-free solar cells [32] as well as their theory-based definition of donor and acceptor strength in conjugated copolymers [33] are of general relevance. Therefore, we assumed that a diversity oriented one-pot methodology could not only provide libraries of various consanguineous 
series of merocyanines, but also could provide access to systematic correlation analyses. Here, we present the methodological extension of our recently reported consecutive SuzukiKnoevenagel three-component synthesis to a broad range of merocyanines, their electronic data by photophysics (absorption and emission spectroscopy) and cyclic voltammetry, as well as several established structure-property relationships by correlation analyses between the compounds' oxidation potentials and their optical band gaps.

\section{Results and Discussion}

\subsection{Synthesis}

Recently, we have established the one-pot Suzuki-Knoevenagel condensation (SuKnoCon) sequence for synthesizing carboxylic acid functionalized merocyanines with tunable donors for DSSC studies [31]. We reasoned that this general, straightforward principle of concatenating Suzuki arylation and Knoevenagel condensation in a consecutive three-component fashion can be particularly favorable for accessing substance libraries of dyes for establishing structure-property relationships based upon correlation analysis (Scheme 1).

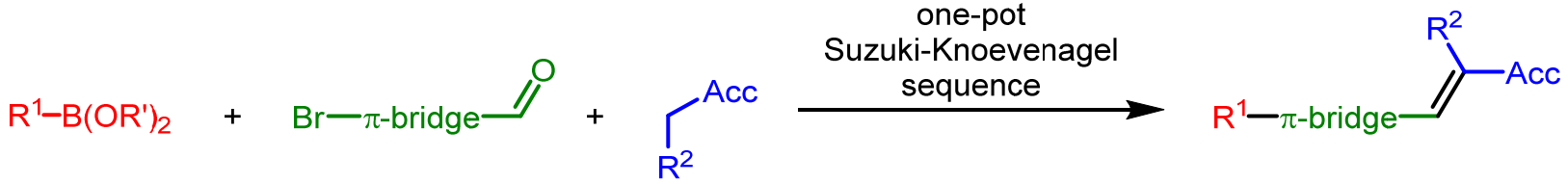

Scheme 1. Multicomponent synthetic concept based upon a Suzuki-Knoevenagel sequence.

As a central bifunctional relay, we chose five different types of bromo aldehydes bearing the two prerequisite functionalities for Suzuki arylation and Knoevenagel condensation and representing $\pi$-bridges ranging from $p$-phenylene (1) over 2,5-thienylene (2) to 4-ocyl 2,5-thienyl (3), 3,6-carbazolylene (4) and 3,7-phenazinylene (5). While the first two possess high lying oxidation potentials, the latter three are increasingly easy to oxidize. The conditions from the sequences were essentially transposed from our previous synthetic study on DSSC dyes [31]. Upon consecutive three-component Suzuki-Knoevenagel synthesis of bromo aldehydes 1-5 with (hetero)aryl boronates or boronic acids $\mathbf{6}$ and methylene active compounds $\mathbf{7}$ in a one-pot fashion five different libraries of merocyanines 8-12, in total 59 compounds, were efficiently synthesized in largely good to excellent yield (Scheme 2). Besides the bromo aldehydes 1-5 the diversity of the libraries relies on sixteen (hetero)aryl boronates or boronic acids 6 and seven methylene active compounds 7 (Scheme 3). While the boronic acid derivatives are electro neutral $(\mathbf{6 a}, \mathbf{6 f}, \mathbf{6 g}, \mathbf{6 h}$, and $\mathbf{6 i})$, electron deficient (6c and $6 \mathrm{~m}$ ) and mostly electron-rich $(6 \mathrm{~b}, 6 \mathrm{~d}, 6 \mathrm{e}, 6 \mathbf{6}-6 \mathbf{1}, 6 \mathrm{n}-6 \mathrm{p})$, the employed methylene active compounds 7 furnish variably strong electron acceptors upon Knoevenagel condensation. All new compounds 8-12, as well as their electronic reference systems 14-17 (vide infra, Scheme 4), were unambiguously assigned in their structure by ${ }^{1} \mathrm{H}$ and ${ }^{13} \mathrm{C}$ NMR spectroscopy, IR spectroscopy and mass spectrometry. The elemental compositions were confirmed by combustion analyses.

The obtained five libraries of $p$-phenylene-bridged (8), thienylene-bridged (9), 4-octyl thienylene-bridged (10), carbazole-bridged systems (11), and phenothiazine-bridged (12) systems containing the merocyanine typical donor- $\pi$-bridge-acceptor motif are depicted in Charts $1-5$.

For electronic comparison and for establishing acceptor parameters (vide infra) phenothiazine aldehyde 13 was reacted by Knoevenagel condensation with methylene active compounds $\mathbf{7 a}, \mathbf{7 c}, \mathbf{7 e}$, and $\mathbf{7 f}$ to give merocyanines $\mathbf{1 4 - 1 7}$ in excellent yield (Scheme 4). 


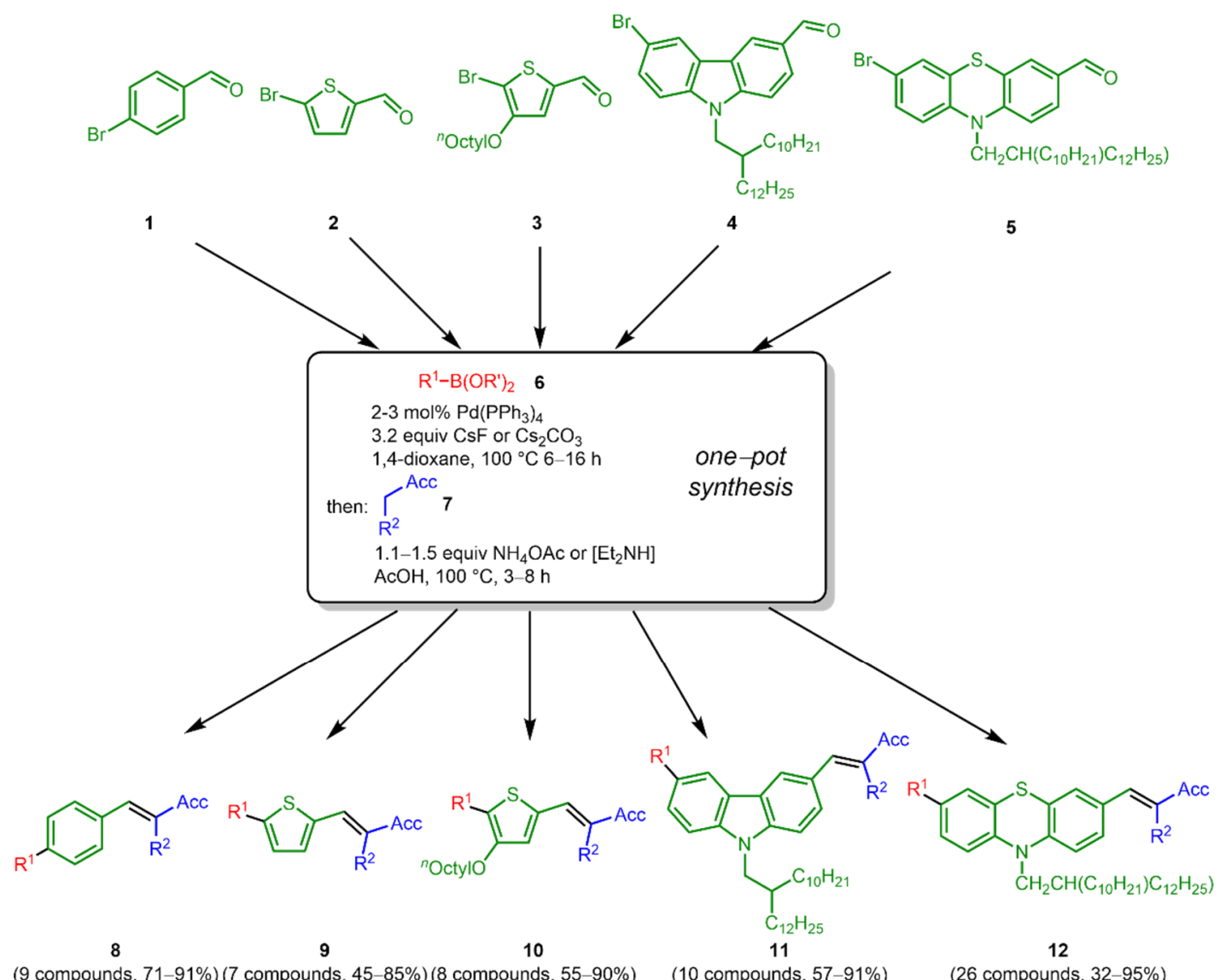

(9 compounds, $71-91 \%)(7$ compounds, $45-85 \%)(8$ compounds, $55-90 \%) \quad$ (10 compounds, $57-91 \%) \quad$ (26 compounds, $32-95 \%)$

Scheme 2. Consecutive three-component Suzuki-Knoevenagel synthesis of merocyanines 8-12.

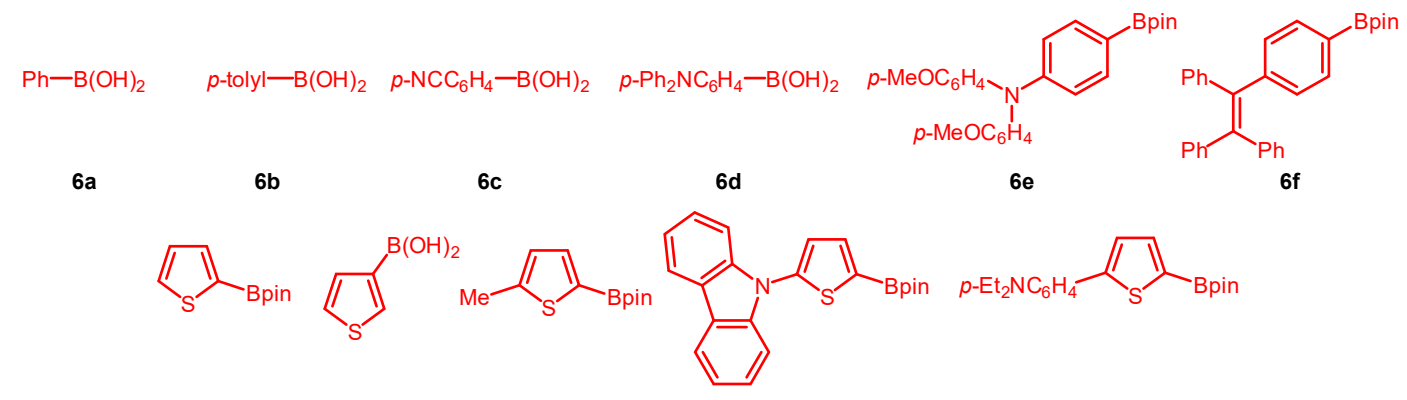

$6 g$

$6 \mathrm{~h}$

$6 \mathbf{i}$

$6 \mathbf{j}$

$6 \mathrm{k}$<smiles>CCCCCCCCCCCc1ccc2c(c1)Sc1cc(Br)ccc1N2CCc1cccc(Br)c1</smiles>

61

$6 \mathrm{~m}$

$6 n$

60<smiles>CN1C(=O)CSC1=S</smiles>

$7 a$<smiles>O=C1CC(=O)c2ccccc21</smiles>

7c<smiles>CCCN1C(=O)CC(=O)N(C(=O)c2ccccc2)C1=O</smiles>

7d<smiles>CC1=NN(c2ccccc2)C(=O)C1</smiles>

$7 e$<smiles>N#CCc1ccc([N+](=O)[O-])cc1</smiles>

$7 f$

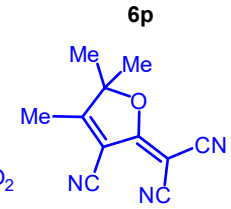

$7 \mathrm{~g}$

Scheme 3. (Hetero)aryl boronates or boronic acids 6 (red) and methylene active compounds 7 (blue) employed in consecutive three-component Suzuki-Knoevenagel syntheses. 


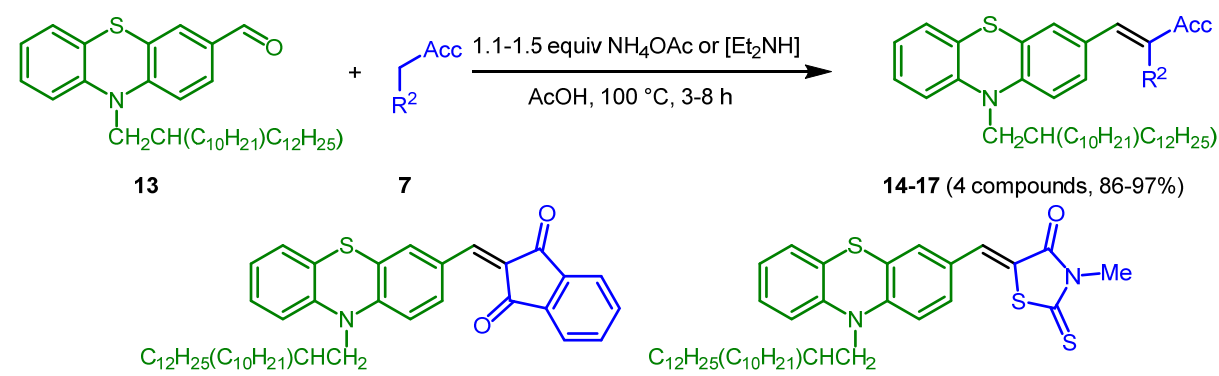<smiles>N#C/C(=C\c1ccc2c(c1)Sc1ccccc1N2)c1ccc([N+](=O)[O-])cc1</smiles>

$\mathrm{C}_{12} \mathrm{H}_{25}\left(\mathrm{C}_{10} \mathrm{H}_{21}\right) \mathrm{CHCH}_{2}$

$16(97 \%)$

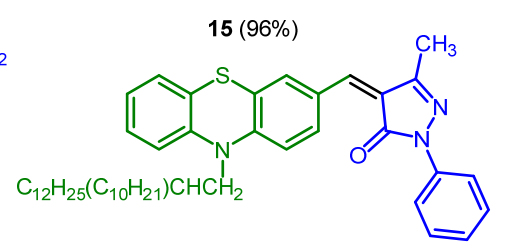

$17(86 \%)$

Scheme 4. Knoevenagel synthesis of merocyanines 14-17.<smiles>CN1C(=O)/C(=C/c2ccc(-c3ccccc3)cc2)SC1=S</smiles>

$8 a$<smiles></smiles>

$8 f$<smiles>O=C1C(=Cc2ccc(-c3ccccc3)cc2)C(=O)c2ccccc21</smiles>

$8 b$<smiles>Cn1cc(-c2ccc(C=C3C(=O)c4ccccc4C3=O)cc2)cn1</smiles>

89<smiles></smiles>

$8 c$<smiles>CC1=NN(c2ccccc2)C(=O)C1=Cc1ccc([18O])cc1</smiles>

$8 d$

$8 e$<smiles>CN1C(=O)/C(=C/c2ccc(-c3ccc4c(c3)Sc3ccccc3N4C)cc2)SC1=S</smiles>

$8 \mathrm{~h}$

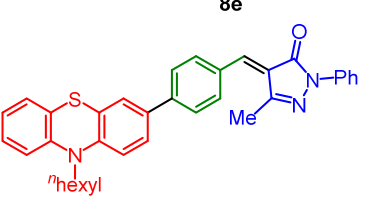

$8 \mathbf{i}$

Chart 1. $p$-Phenylene-bridged systems 8.

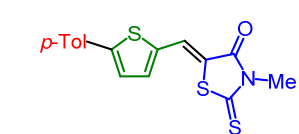

$9 a$

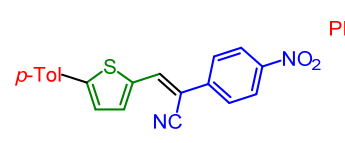

$9 e$<smiles>O=C1C(=Cc2ccc(C(F)F)s2)C(=O)c2ccccc21</smiles>

9b<smiles>CN1C(=O)/C(=C/c2ccc(-c3ccc(N)cc3)s2)SC1=S</smiles>

9f

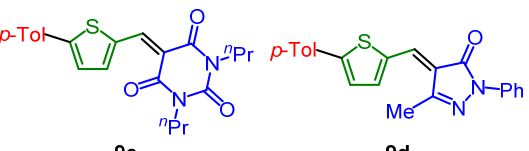

$9 \mathrm{c}$

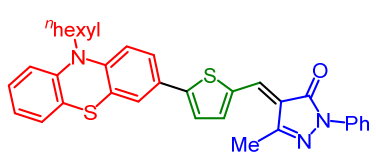

$9 \mathrm{~g}$

Chart 2. Thienylene-bridged systems 9.

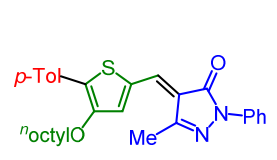

$10 \mathrm{a}$

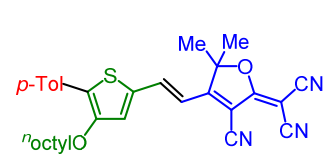

10b

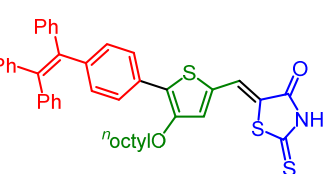

$10 \mathrm{c}$

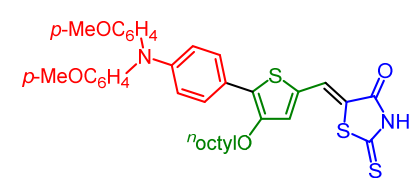

$10 \mathrm{~d}$

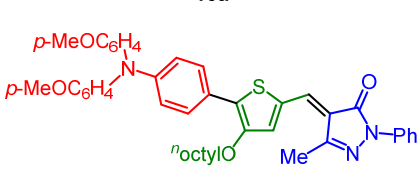

$10 \mathrm{e}$

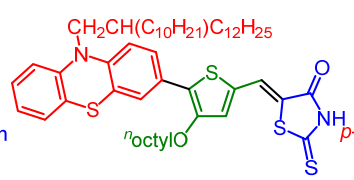

$10 f$

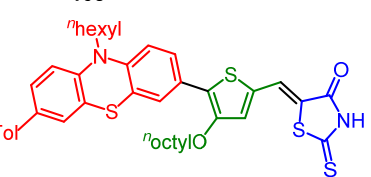

$10 \mathrm{~g}$

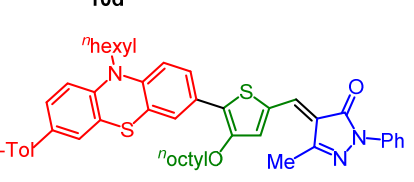

$10 \mathrm{~h}$

Chart 3. 4-Octyl thienylene-bridged systems 10. 


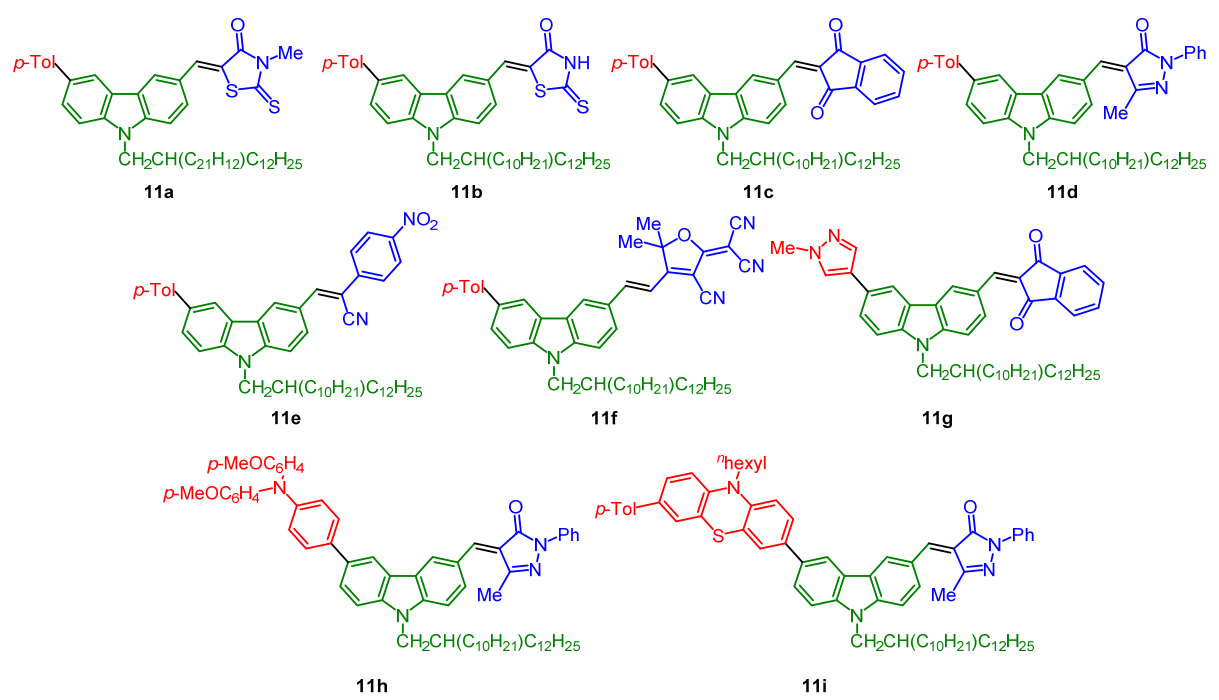

Chart 4. Carbazole-bridged systems 11.

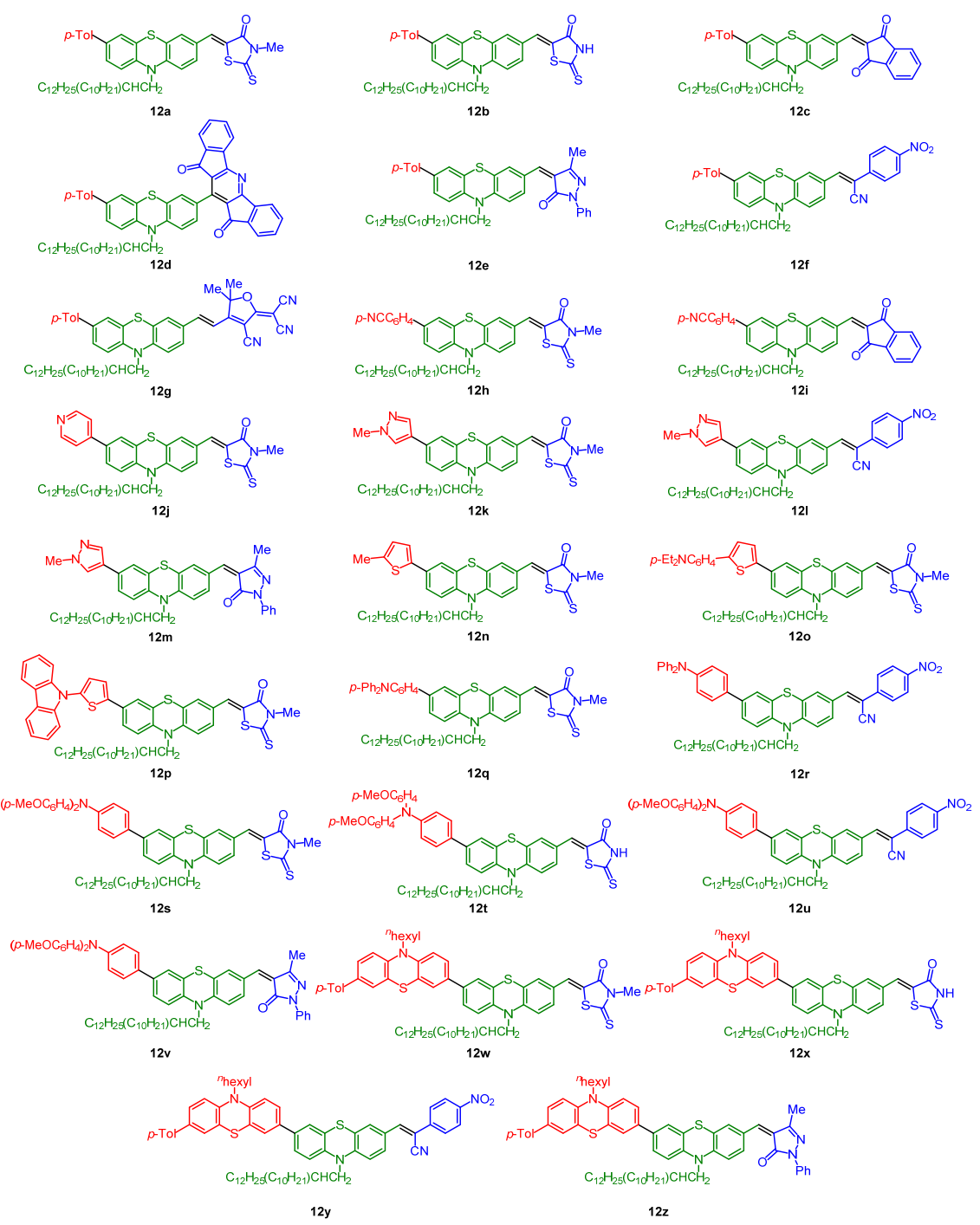

Chart 5. Phenothiazine-bridged systems 12. 


\subsection{Electronic Characteristics}

The electronic properties of the merocyanines 8-12 were determined by recording the absorption and emission spectra as well as the cyclic voltammograms in the anodic regions (oxidations) (Table 1). While oxidation potentials reflect a property of the electronic ground state of the chromophores, their absorption and emission behavior correlate to the photonic transitions from the electronic ground to the excited states and back. The oxidation potential of the compounds predominantly depends on the presence of a readily oxidizable moiety in the $\pi$-bridge, such as 4-octyl thienyl, carbazole or phenothiazine units, or in the substituent $R^{1}$. As in our previous study [31], we calculated the half-wave potentials $E_{1 / 2}$ referenced against the normal hydrogen electrode by adding $0.2 \mathrm{~V}$ to the measured value of $E_{0}$ (against $\mathrm{Ag} / \mathrm{AgCl}$ ). The first oxidations were mostly obtained as electrochemically reversible waves in a range from 0.79 to $1.71 \mathrm{~V}$. For irreversible potentials, only the anodic peak potential was documented. The obtained merocyanines display longest wavelength absorption maxima $\lambda_{\text {max,abs }}$ covering a broad part of the $U V / V$ is spectrum ranging from 367 to $580 \mathrm{~nm}$. As seen from the molar decadic extinction coefficients, these transitions are quite intense and account to charge transfer character from the donor to the acceptor moiety, as previously corroborated for phenothiazine-based DSSC merocyanines [31]. In addition, most of the compounds reveal emission maxima $\lambda_{\mathrm{max}, \mathrm{em}}$ ranging from 412 to $668 \mathrm{~nm}$, which were not quantified due to very variable intensity. Nevertheless, Stokes shifts $\Delta \tilde{v}$, i.e., energy differences between longest wavelength absorption maxima and emission maxima, as an indicator of changes in the electronic structure upon photonic excitation from the ground state to the vibrationally relaxed excited states were calculated in a range between 1200 and $8000 \mathrm{~cm}^{-1}(0.147-0.990 \mathrm{eV})$. In addition, from the absorption and emission maxima the optical band gap, i.e., the $E_{0-0}$ transition, was estimated by the arithmetic average of the corresponding and ranging from 2.083 to $3.197 \mathrm{eV}$.

Table 1. Selected electronic properties of Merocyanines 8-12 (recorded in $\mathrm{CH}_{2} \mathrm{Cl}_{2}, \mathrm{~T}=298 \mathrm{~K}$ ).

\begin{tabular}{|c|c|c|c|c|c|}
\hline Compound & $\begin{array}{l}E_{1 / 2} \text { vs. NHE } \\
{\left[\mathrm{V}^{1}{ }^{1}\right.}\end{array}$ & $\begin{array}{c}\lambda_{\max , \mathrm{abs}} \\
{[\mathrm{nm}]\left(\varepsilon\left[\mathrm{M}^{-1} \mathrm{~cm}^{-1}\right]\right)}\end{array}$ & $\begin{array}{c}\lambda_{\text {max }, \text { em }} \\
{[\mathrm{nm}]^{2}}\end{array}$ & $\begin{array}{l}\text { Stokes Shift } \Delta \tilde{v} \\
{\left[\mathrm{~cm}^{-1}\right]([\mathrm{eV}])^{3}}\end{array}$ & $\begin{array}{c}E_{0-0} \\
{[\mathrm{eV}]^{4}}\end{array}$ \\
\hline $8 a$ & $>2.00$ & $293(18,000), 396(42,000)$ & 451 & $3100(0.382)$ & 2.943 \\
\hline $8 b$ & $>2.00$ & $261(30,000), 302(20,000), 384(24,000), 438(26,000)$ & 462 & $1200(0.147)$ & 2.760 \\
\hline $8 c$ & $>2.00$ & $293(14,000), 398(44,000)$ & 470 & $3900(0.478)$ & 2.879 \\
\hline $8 d$ & $>2.00$ & $294(15,000), 364(23,000)$ & 513 & $8000(0.990)$ & 2.914 \\
\hline $8 e$ & 1.57 (irrev.) & $250(25,000), 367(35,000)$ & 412 & $3000(0.369)$ & 3.197 \\
\hline $8 f$ & $>2.00$ & $296(15,000), 402(38,000)$ & 463 & $3300(0.407)$ & 2.820 \\
\hline $8 g$ & 1.55 (irrev.) & $258(22,000), 265(21,000), 403(38,000)$ & 486 & $4200(0.526)$ & 2.866 \\
\hline $8 \mathrm{~h}$ & $0.95,1.66$ & $261(30,000), 302(20,000), 384(24,000), 438(26,000)$ & 555 & $4800(0.597)$ & 2.535 \\
\hline $8 \mathbf{i}$ & $0.93,1.61$ & $259(35,000), 334(20,000), 442(15,000)$ & 561 & $4800(0.596)$ & 2.510 \\
\hline 9a & 1.60 (irrev.) & $289(12,000), 313(18,000), 451(48,000)$ & 514 & $2700(0.337)$ & 2.583 \\
\hline $9 b$ & 1.76 & $267(23,000), 278(14,000), 304(8000), 450(43,000)$ & 513 & $2700(0.339)$ & 2.588 \\
\hline $9 \mathrm{c}$ & $>2.00$ & $275(13,000), 301(4000), 434(41,000)$ & 502 & $3100(0.387)$ & 2.666 \\
\hline $9 d$ & 1.61 (irrev.) & $259(25,000), 307(5000), 421(37,000)$ & 481 & $3000(0.368)$ & 2.764 \\
\hline $9 e$ & 1.71 & $257(43,000), 387(54,000)$ & 525 & $6800(0.843)$ & 2.785 \\
\hline 9f & 1.13 (irr) & $305(27,000), 352(15,000), 499(42,000)$ & 632 & $4200(0.523)$ & 2.225 \\
\hline $9 g$ & $0.93,1.61$ & $256(40,000), 304(13,000), 379(17,000), 490(28,000)$ & 604 & $3900(0.478)$ & 2.294 \\
\hline $10 a$ & 1.45 & $259(21,000), 337(9000), 462(31,000)$ & -5 & -5 & - \\
\hline $10 \mathrm{~b}$ & 1.46 & $270(13,000), 324(21,000), 550(26,000)$ & 650 & $2800(0.347)$ & 2.083 \\
\hline 10c & $1.38,1.57$ & $289(22,000), 314(22,000), 349(25,000), 500(50,000)$ & 594 & $3200(0.393)$ & 2.286 \\
\hline 10d & $0.87,1.38$ & $305(27,000), 351(23,000), 550(40,000)$ & -5 & -5 & - \\
\hline $10 \mathrm{e}$ & $0.87,1.43$ & $256(28,000), 310(21,000), 401(6000), 556(41,000)$ & -5 & -5 & - \\
\hline $10 f$ & $0.93,1.46$ & $\begin{array}{c}262(21,000), 313(19,000), 347(17,000), 419(11,000), 523 \\
(33,000)\end{array}$ & -5 & -5 & - \\
\hline $10 \mathrm{~g}$ & $0.86,1.40$ & $\begin{array}{c}274(37,000), 310(26,000), 345(20,000), 420(12,000), 527 \\
(37000)\end{array}$ & -5 & -5 & - \\
\hline
\end{tabular}


Table 1. Cont.

\begin{tabular}{|c|c|c|c|c|c|}
\hline Compound & $\begin{array}{c}E_{1 / 2} \text { vs. NHE } \\
{\left[\mathrm{V}^{1}\right.}\end{array}$ & $\begin{array}{c}\lambda_{\text {max, abs }} \\
{[\mathrm{nm}]\left(\varepsilon\left[\mathbf{M}^{-1} \mathrm{~cm}^{-1}\right]\right)}\end{array}$ & $\begin{array}{c}\lambda_{\text {max,em }} \\
{[\mathrm{nm}]^{2}}\end{array}$ & $\begin{array}{l}\text { Stokes Shift } \Delta \tilde{v} \\
{\left[\mathrm{~cm}^{-1}\right]([\mathrm{eV}])^{3}}\end{array}$ & $\begin{array}{c}E_{0-0} \\
{[\mathrm{eV}]^{4}}\end{array}$ \\
\hline $10 \mathrm{~h}$ & $0.86,1.51$ & $258(51,000), 319(20,000), 417(11,000), 533(30,000)$ & $-{ }^{5}$ & -5 & - \\
\hline $11 \mathrm{a}$ & 1.44 & $\begin{array}{c}253(41,000), 262(40,000), 298(41,000), 354(15,000), 453 \\
(44,000)\end{array}$ & 526 & $3100(0.380)$ & 2.549 \\
\hline $11 b$ & 1.44 & $\begin{array}{c}253(34,000), 262(35,000), 286(35,000), 356(13,000), 453 \\
(39,000)\end{array}$ & 530 & $3200(0.398)$ & 2.541 \\
\hline 11c & 1.46 & $253(55,000), 285(46,000), 347(21,000), 464(52,000)$ & 561 & $3700(0.462)$ & 2.443 \\
\hline 11d & 1.45 & $256(44,000), 280(40,000), 342(22,000), 428(29,000)$ & 526 & $4400(0.540)$ & 2.629 \\
\hline 11e & 1.44 & $260(37,000), 284(40,000), 344(19,000), 431(31,000)$ & 558 & $5200(0.655)$ & 2.552 \\
\hline $11 f$ & 1.44 & $254(40,000), 290(32,000), 366(13,000), 530(45,000)$ & 628 & $2900(0.365)$ & 2.159 \\
\hline $11 \mathrm{~g}$ & 1.34 & $281(34,000), 303(21,000), 352(19,000), 467(43,000)$ & 583 & $4300(0.529)$ & 2.393 \\
\hline $11 \mathrm{~h}$ & $0.82,1.38$ & $252(42,000), 307(39,000), 329(40,000), 425(23,000)$ & -5 & -5 & - \\
\hline $11 \mathbf{i}$ & $0.81,1.39$ & $286(69,000), 341(28,000), 424(26,000)$ & -5 & $-{ }^{5}$ & - \\
\hline $12 a$ & $1.05,1.68$ & $271(34,000), 302(27,000), 361(20,000), 482(24,000)$ & 655 & $5500(0.680)$ & 2.235 \\
\hline $12 b$ & $1.05,1.68$ & $271(30,000), 303(24,000), 358(21,000), 483(25,000)$ & 658 & $5500(0.683)$ & 2.228 \\
\hline $12 c$ & $1.05,1.69$ & $268(46,000), 309(23,000), 338(25,000), 512(27,000)$ & 685 & $4900(0.612)$ & 2.118 \\
\hline $12 e$ & $1.05,1.70$ & $260(49,000), 311(35,000), 487(25,000)$ & 638 & $4900(0.603)$ & 2.247 \\
\hline $12 f$ & $1.03,1.69$ & $271(29,000), 319(27,000), 472(21,000)$ & 580 & $4000(0.490)$ & 2.384 \\
\hline $12 \mathrm{~g}$ & $1.05,1.69$ & $\begin{array}{c}267(34,000), 320(13,000), 392(20,000), 410(19,000), 580 \\
(34,000)\end{array}$ & $-{ }^{5}$ & -5 & - \\
\hline $12 \mathrm{~h}$ & -6 & $280(27,000), 307(28,000), 358(26,000), 473(28,000)$ & 641 & $5500(0.688)$ & 2.280 \\
\hline $12 \mathbf{i}$ & 1.13 & $268(35,000), 331(29,000), 362(21,000), 504(28,000)$ & 668 & $4900(0.605)$ & 2.160 \\
\hline $12 j$ & 1.17 & $277(24,000), 304(29,000), 358(23,000), 472(29,000)$ & 636 & $5500(0.678)$ & 2.290 \\
\hline $12 k$ & 1.01 & $267(32,000), 297(25,000), 363(21,000), 484(25,000)$ & 660 & $5500(0.684)$ & 2.222 \\
\hline 121 & $1.03,1.69$ & $270(29,000), 332(25,000), 474(21,000)$ & 580 & $4000(0.478)$ & 2.379 \\
\hline $12 \mathrm{~m}$ & $1.00,1.61$ & $261(45,000), 312(27,000), 489(21,000)$ & 614 & $4200(0.517)$ & 2.280 \\
\hline $12 n$ & $1.03,1.57$ & $303(29,000), 360(23,000), 483(23,000)$ & 640 & $5100(0.630)$ & 2.254 \\
\hline 120 & $0.79,1.01$ & $305(23,000), 371(49,000), 492(28,000)$ & -5 & -5 & - \\
\hline $12 p$ & $1.06,1.33$ & $293(18,000), 396(42,000)$ & 651 & $5400(0.674)$ & 2.243 \\
\hline $12 \mathrm{r}$ & $0.98,1.20$ & $261(30,000), 302(20,000), 384(24,000), 438(26,000)$ & $-{ }^{5}$ & -5 & - \\
\hline $12 \mathrm{~s}$ & $0.86,1.12$ & $293(14,000), 398(44,000)$ & 629 & $4600(0.565)$ & 2.255 \\
\hline $12 t$ & $0.86,1.80$ & $294(15,000), 364(23,000)$ & 634 & $4500(0.560)$ & 2.237 \\
\hline $12 u$ & $0.86,1.11$ & $250(25,000), 367(35,000)$ & -5 & -5 & - \\
\hline $12 v$ & $\begin{array}{l}0.85,1.11,1.67 \\
1.79\end{array}$ & $296(15,000), 402(38,000)$ & $-{ }^{5}$ & $-{ }^{5}$ & - \\
\hline $12 w$ & $0.85,1.12$ & $258(22,000), 265(21,000), 403(38,000)$ & 658 & $5400(0.667)$ & 2.220 \\
\hline $12 x$ & $0.86,1.14,1.47$ & $261(30,000), 302(20,000), 384(24,000), 438(26,000)$ & 641 & $4700(0.586)$ & 2.229 \\
\hline $12 y$ & $0.85,1.17$ & $259(35,000), 334(20,000), 442(15,000)$ & 657 & $4900(0.613)$ & 2.195 \\
\hline $12 z$ & $\begin{array}{c}0.85,1.11,1.66 \\
1.81\end{array}$ & $289(12,000), 313(18,000), 451(48,000)$ & -5 & -5 & - \\
\hline 14 & 1.10 & $267(23,000), 278(14,000), 304(8000), 450(43,000)$ & 674 & $5000(0.626)$ & 2.154 \\
\hline 15 & 1.09 & $275(13,000), 301(4000), 434(41,000)$ & 642 & $5700(0.696)$ & 2.281 \\
\hline 16 & $1.08,1.76$ & $259(25,000), 307(5000), 421(37,000)$ & 565 & $3900(0.490)$ & 2.441 \\
\hline 17 & 1.09 & $257(43,000), 387(54,000)$ & 640 & $5400(0.668)$ & 2.273 \\
\hline
\end{tabular}

${ }^{1}$ Recorded in $\mathrm{CH}_{2} \mathrm{Cl}_{2}, \mathrm{~T}=293 \mathrm{~K}, v=100 \mathrm{mV} \cdot \mathrm{s}^{-1}$, electrolyte: [ $\left.\mathrm{Bu}_{4} \mathrm{~N}\right]\left[\mathrm{PF}_{6}\right]$, Pt-working electrode, Pt-counter electrode, $\mathrm{Ag} / \mathrm{AgCl}$ reference electrode; $E_{0}=\left(E_{\mathrm{pa}}+E_{\mathrm{pc}}\right) / 2$ referenced to $[\mathrm{Fc}] /[\mathrm{Fc}]^{+}=0.45 \mathrm{~V}[34] ; E_{1 / 2}=E_{0}+0.20 \mathrm{~V}$ (referenced against NHE). ${ }^{2} \lambda_{\text {exc }}$ at longest wavelength maximum. ${ }^{3} \Delta \tilde{v}=1 / \lambda_{\max , a b s}-1 / \lambda_{\max , \mathrm{em}}\left[\mathrm{cm}^{-1}\right]\left(\Delta \tilde{v}=1241 \cdot\left(1 / \lambda_{\max , a b s}-1 / \lambda_{\max , \mathrm{em}}\right)[\mathrm{eV}]\right) .{ }^{4} E_{0-0}$ : the energy of the hypothetical $0-0$-transition determined from the intersection of the absorption and emission band, i.e., $E_{0-0}=\left[1241 \cdot\left(1 / \lambda_{\max , \mathrm{abs}}+1 / \lambda_{\max , \mathrm{em}}\right)\right] / 2$ [eV] (wavelength inserted as nanometer value). ${ }^{5}$ No detectable emission. ${ }^{6}$ The potential was not determined.

\subsection{Correlation Analyses of the Electronic Properties}

The extensive merocyanine libraries 8-12 encouraged us to take a closer look on a semiempirical physical organic correlation analysis. Correlations between clearly assignable oxidation potentials and $E_{0-0}$ transitions should provide an insight into structure-property relationships of this class of compounds. Already visible to the naked eye, the nature of the $\pi$-bridge between donor and acceptor moieties, as seen for four phenothiazine- $N$-phenyl 
methyl pyrazolone merocyanines $17,8 \mathbf{i}, \mathbf{9 g}$ and $10 \mathrm{~h}$, causes significant differences in the electronic properties (Figure 1).
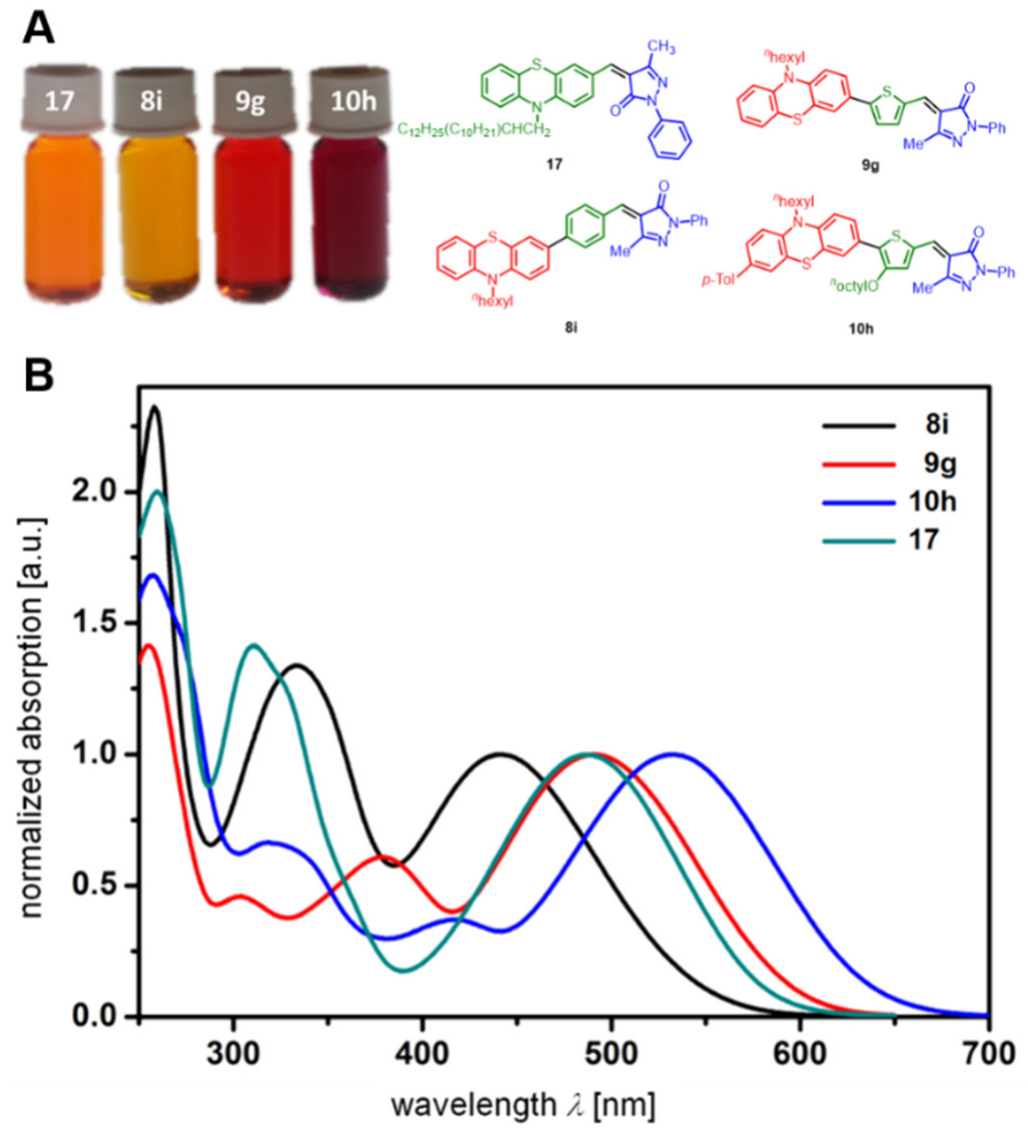

Figure 1. Comparison of four phenothiazine- $N$-phenyl methyl pyrazolone merocyanines with variable $\pi$-bridging. (A) Solutions of compounds $17,8 \mathbf{i}, \mathbf{9 g}$ and $\mathbf{1 0 h}$ (from left to right) under daylight $\left(c=10^{-3} \mathrm{M}\right)$. (B) Normalized UV/Vis spectra of compounds $\mathbf{1 7}, \mathbf{8 i}, \mathbf{9 g}$ and $\mathbf{1 0 h}$ (recorded in dichloromethane, $\left.c=10^{-3} \mathrm{M}, \mathrm{T}=298 \mathrm{~K}\right)$.

For establishing a set of structure-property relationships we considered four classes of acceptors, i.e., 3-methyl-4-oxo-2-thioxothiazolidin-5-ylidene (from condensation of 7a), 1,3dioxo-1,3-dihydro-2H-inden-2-ylidene (from condensation of 7c), 3-methyl-5-oxo-1-phenyl1,5-dihydro-4H-pyrazol-4-ylidene (from condensation of 7e), cyano(4-nitrophenyl)-methylene (from condensation of $\mathbf{7 f}$ ), and four classes of donor bridge systems of the merocyanine series 9 (5-( $p$-tolyl)thien-2-yl)), 11 (6-(p-tolyl)carbzol-3-yl $)), 12$ (7-( $p$-tolyl)phenothiazin-3-yl) as well as the reference chromophores 14-17 (phenothiazin-3-yl) (Table 2).

A qualitative look at the electronic data $\left(E_{1 / 2}, \lambda_{\text {max,abs }}, \lambda_{\text {max,em, }}\right.$, Stokes shift $\Delta \tilde{v}$, and $\left.E_{0-0}\right)$ shows that the oxidation potential $E_{1 / 2}$ reflecting the donor strength is affected by the acceptor strength and a qualitative alignment acceptors with decreasing strength concomitantly correlates with an increasing optical band gap $E_{0-0}$ (Figure 2). In the same trend absorption and emission bands are blue shifted (to shorter wavelength) with decreasing acceptor strength. For the unperturbed reference merocyanines 14-17, the plots of $\lambda_{\text {max,abs, }}$ $\lambda_{\text {max,em }}$ and $E_{0-0}$ (as energies in $\mathrm{eV}$ ) against $E_{1 / 2}$ (in Volt) give reasonably good linear correlations $\left(\mathrm{r}^{2}>0.90\right)$ (for details on correlation analyses, see Supplementary Material). Since the series 9, 11 and 12, maintaining the constant donor moiety while varying the acceptor parts, give poor correlations, we instead considered variations of the donor parts while maintaining the corresponding acceptors constant as grouped in Table 2. 
Table 2. Selected electronic properties of merocyanine series $\mathbf{9 , 1 1 , 1 2}$, and 14-17 employed in the correlation analyses.

\begin{tabular}{|c|c|c|c|c|c|c|}
\hline \multirow[b]{2}{*}{ Acceptor } & $9 a, 9 b, 9 d, 9 e$ & \multicolumn{2}{|c|}{$11 \mathrm{a}, 11 \mathrm{c}, 11 \mathrm{~d}, 11 \mathrm{e}$} & $\begin{array}{l}{ }^{2} \mathrm{CH}\left(\mathrm{C}_{21} \mathrm{H}\right. \\
{ }_{14-17}\end{array}$ & \multicolumn{2}{|c|}{ 12a, 12c, 12e, $12 f$} \\
\hline & & $E_{1 / 2}[V]$ & $\lambda_{\max , \mathrm{abs}}$ & $\begin{array}{c}\lambda_{\max , \mathrm{em}} \\
{[\mathrm{eV}]}\end{array}$ & $\Delta \tilde{v}[\mathrm{eV}]$ & $E_{0-0}[e V]$ \\
\hline & $9 a$ & 1.66 & 2.752 & 2.414 & 0.337 & 2.583 \\
\hline & $11 a$ & 1.44 & 2.740 & 2.359 & 0.380 & 2.549 \\
\hline & $12 \mathbf{a}$ & 1.05 & 2.575 & 1.895 & 0.680 & 2.235 \\
\hline & 15 & 1.09 & 2.629 & 1.933 & 0.696 & 2.281 \\
\hline & $9 b$ & 1.76 & 2.758 & 2.419 & 0.339 & 2.588 \\
\hline & 11c & 1.46 & 2.675 & 2.212 & 0.462 & 2.443 \\
\hline & $12 \mathrm{c}$ & 1.05 & 2.424 & 1.812 & 0.612 & 2.118 \\
\hline & 14 & 1.10 & 2.467 & 1.841 & 0.626 & 2.154 \\
\hline & $9 d$ & 1.61 & 2.948 & 2.580 & 0.368 & 2.764 \\
\hline & $11 d$ & 1.45 & 2.899 & 2.359 & 0.540 & 2.629 \\
\hline & $12 e$ & 1.05 & 2.548 & 1.945 & 0.603 & 2.246 \\
\hline & 17 & 1.09 & 2.607 & 1.939 & 0.668 & 2.273 \\
\hline & $9 e$ & 1.71 & 3.207 & 2.364 & 0.843 & 2.785 \\
\hline & 11e & 1.44 & 2.879 & 2.224 & 0.655 & 2.552 \\
\hline & $12 f$ & 1.03 & 2.629 & 2.140 & 0.490 & 2.384 \\
\hline CN & 16 & 1.08 & 2.686 & 2.196 & 0.489 & 2.441 \\
\hline
\end{tabular}<smiles>C=C1C(=O)c2ccccc2C1=O</smiles><smiles>C=C1SC(=S)N(C)C1=O</smiles><smiles>C=C1C(=O)N(c2ccccc2)N=C1C</smiles><smiles>C=C(C#N)c1ccc([N+](=O)[O-])cc1</smiles>

\section{$E_{1 / 2}$ \\ $E_{0-0}$ \\ $\lambda_{\max , a b s}$ \\ $\lambda_{\text {max }, e m}$}

Figure 2. Qualitative acceptor strength decreasing from left to right and their influence on selected electronic data $\left(E_{1 / 2}, E_{0-0}, \lambda_{\max , \text { abs }}\right.$ and $\left.\lambda_{\max , \mathrm{em}}\right)$.

The consanguineous acceptor series of 3-methyl-4-oxo-2-thioxothiazolidin-5-ylidene, 1,3-dioxo-1,3-dihydro-2H-inden-2-ylidene, 3-methyl-5-oxo-1-phenyl-1,5-dihydro-4H-pyrazol4-ylidene and cyano(4-nitrophenyl)-methylene derivatives gave good to excellent linear correlations for the plots of $\lambda_{\max , a b s}, \lambda_{\max , \mathrm{em}}$, Stokes shift $\Delta \tilde{v}$ and $E_{0-0}$ (as energies in $\mathrm{eV}$ ) against $E_{1 / 2}$ (in Volt) $\left(\mathrm{r}^{2}>0.90\right)$ (for details on correlation analyses, see Supplementary Material). In particular, the excellent linear correlations $\left(\mathrm{r}^{2}=0.943-0.999\right)$ between the oxidation potential $E_{1 / 2}$ and the optical band gap $E_{0-0}$ (Table 3)) suggest that the electronic ground state property, i.e., oxidation potential, affects the photonic property, i.e., band gap, and could lead to a more general correlation beyond consanguineous chromophore series. Therefore, we expanded the basis of chromophores to a total of 24 (for correlation analyses, see Supplementary Information). An attempt to establish a direct relation between $E_{1 / 2}$ and $E_{00}$ gave only a poor linear correlation $\left(r^{2}=0.7338\right)$. 
Table 3. Linear correlation analyses of the consanguineous acceptor series.

\begin{tabular}{|c|c|c|}
\hline Acceptor & Linear Correlation Equations $E_{0-0}$ vs. $E_{1 / 2}$ & Correlation Coefficient \\
\hline & $E_{0-0}=1.41368+0.67953 \cdot E_{1 / 2}[\mathrm{eV}]$ & $\mathrm{r}^{2}=0.98716$ \\
\hline & $E_{0-0}=1.25336+0.94225 \cdot E_{1 / 2}[\mathrm{eV}]$ & $\mathrm{r}^{2}=0.99896$ \\
\hline & $E_{0-0}=1.62924+0.59758 \cdot E_{1 / 2}[\mathrm{eV}]$ & $\mathrm{r}^{2}=0.94309$ \\
\hline & $E_{0-0}=1.83274+0.53835 \cdot E_{1 / 2}[\mathrm{eV}]$ & $\mathrm{r}^{2}=0.94765$ \\
\hline
\end{tabular}

However, plotting $E_{0-0}$ vs. the two parameters $E_{1 / 2}$ and $\lambda_{\max , \mathrm{em}}$ representing both ground and excited state energetics give a quite good planar correlation $\left(\mathrm{r}^{2}=0.93504\right)$, were the slopes indicate that the emission $\lambda_{\text {max,em }}$ contributes to a larger extent than the oxidation potentials (Figure 3). While the oxidation potential represents an electronic ground state parameter the emission, mostly resulting from radiative deactivation of the vibrationally relaxed excited state $S_{1}$ depends on the electronic structure of the excited state. For merocyanines typical are highly polar excited states, which attribute for a distinct degree of charge transfer character. This planar correlation now allows for a series of merocyanines to predict optical band gaps from first oxidation potentials and emission maxima.

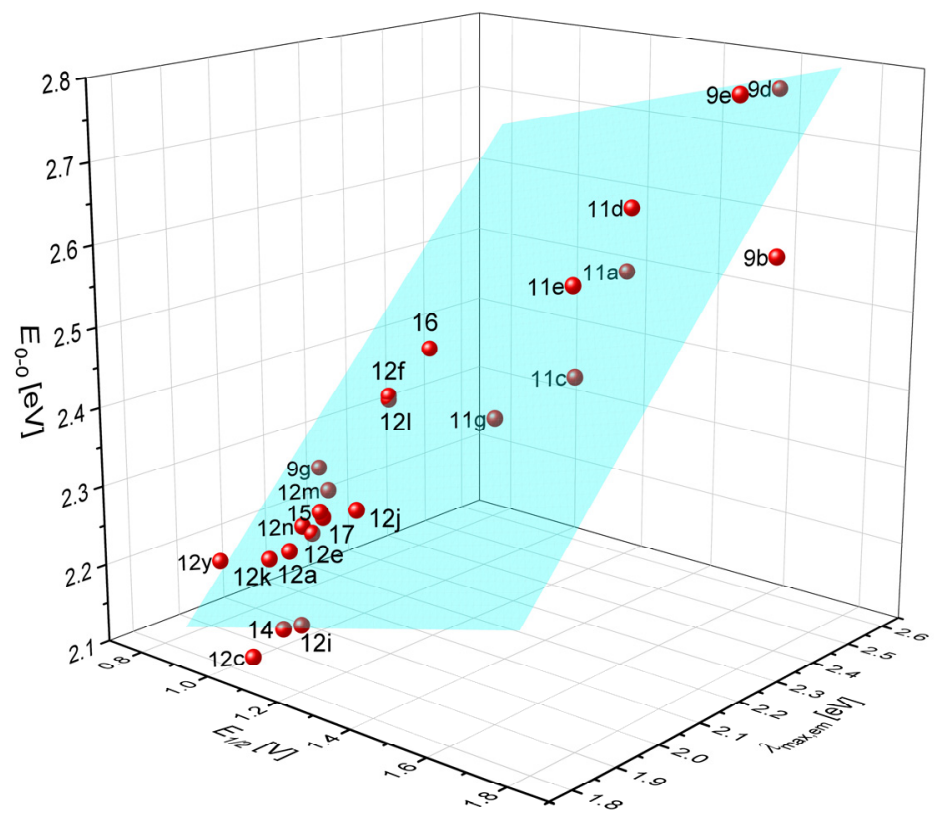

Figure 3. Correlation of the optical band gap $E_{0-0}$ vs. oxidation potential $E_{1 / 2}$ and emission maximum $\lambda_{\text {max }, \mathrm{em}}: E_{0-0}=0.147 \cdot E_{1 / 2}+0.7019 \cdot \lambda_{\max , \mathrm{em}}+0.7296 ; \mathrm{r}^{2}=0.93504$. 


\section{Materials and Methods}

All synthetic details on the preparation as well as the ${ }^{1} \mathrm{H}$ and ${ }^{13} \mathrm{C}$ NMR spectra of the series $8,9,10,11$ and 12 , and the reference chromophores $14-17$ are compiled in the Supporting Information.

\section{Synthesis of Compound $\mathbf{9} \mathbf{d}$ by Coupling-Condensation One-Pot Synthesis (Typical Procedure)}

4-Methylphenylboronic acid (6b) (150 mg, $1.10 \mathrm{mmol})$, 5-bromothiophene-2-carbaldehyde (2) $(190 \mathrm{mg}, 1.00 \mathrm{mmol})$, cesium fluoride $(486 \mathrm{mg}, 3.20 \mathrm{mmol})$ and tetrakis(triphenylphosphane) palladium(0) $(24 \mathrm{mg}, 0.02 \mathrm{mmol})$ were placed in a Schlenk flask with magnetic stir bar under nitrogen and dry 1,4-dioxane $(4 \mathrm{~mL})$ were added. The solution was heated to $100{ }^{\circ} \mathrm{C}$ under reflux for $8 \mathrm{~h}$. After cooling to room temp acetic acid $(2 \mathrm{~mL}), 5$-methyl-2-phenyl2,4-dihydro-3H-pyrazol-3-one (7e) $(192 \mathrm{mg}, 1.10 \mathrm{mmol})$, and 1 drop of diethylamine was added to the reaction mixture. This mixture was heated under nitrogen to $95{ }^{\circ} \mathrm{C}$ under reflux for $4 \mathrm{~h}$. An intensive orange red solution was formed. After cooling to room temp the reaction mixture was diluted with dichloromethane $(30 \mathrm{~mL})$ and the organic layer was washed with distilled water until the aqueous phase did not smell similar to acetic acid. The combined aqueous phases were extracted with dichloromethane and the combined organic layers were dried (anhydrous magnesium sulfate) and the solvents were removed in vacuo. The residue was adsorbed on celite ${ }^{\circledR}$ and purified by flash chromatography on silica gel ( $n$-hexane/acetone 10:1) to furnish after drying under vacuo compound $9 \mathbf{d}$ $(290 \mathrm{mg}, 81 \%)$ as a red solid, $\mathrm{Mp} 133^{\circ} \mathrm{C} . \mathrm{R}_{f}(n$-hexane/acetone 4:1) $=0.27$.

${ }^{1} \mathrm{H}$ NMR $\left(600 \mathrm{MHz}\right.$, acetone- $\left.\mathrm{d}_{6} / \mathrm{CS}_{2}\right): \delta 2.35(\mathrm{~s}, 3 \mathrm{H}), 2.41(\mathrm{~s}, 3 \mathrm{H}), 7.41\left(\mathrm{tt},{ }^{3} \mathrm{~J}=\right.$ $\left.7.4 \mathrm{~Hz},{ }^{4} \mathrm{~J}=1.1 \mathrm{~Hz}, 1 \mathrm{H}\right), 7.27-7.31(\mathrm{~m}, 2 \mathrm{H}), 7.37-7.42(\mathrm{~m}, 2 \mathrm{H}), 7.56\left(\mathrm{~d},{ }^{3} \mathrm{~J}=4.0 \mathrm{~Hz}\right.$, $1 \mathrm{H}), 7.68-7.72(\mathrm{~m}, 2 \mathrm{H}), 7.86(\mathrm{~s}, 1 \mathrm{H}), 8.05-8.08(\mathrm{~m}, 2 \mathrm{H}), 8.11\left(\mathrm{~d},{ }^{3} \mathrm{~J}=4.0 \mathrm{~Hz}, 1 \mathrm{H}\right) .{ }^{13} \mathrm{C}$ NMR $\left(150 \mathrm{MHz}\right.$, acetone- $\left.\mathrm{d}_{6} / \mathrm{CS}_{2}\right): \delta 13.3\left(\mathrm{CH}_{3}\right), 21.6\left(\mathrm{CH}_{3}\right), 118.7(\mathrm{CH}), 122.1\left(\mathrm{C}_{\text {quat }}\right)$, $124.7(\mathrm{CH}), 124.9(\mathrm{CH}), 127.1(\mathrm{CH}), 129.3(\mathrm{CH}), 130.7(\mathrm{CH}), 131.6\left(\mathrm{C}_{\text {quat }}\right), 136.7\left(\mathrm{C}_{\text {quat }}\right)$, 137.2 (CH), $139.9\left(\mathrm{C}_{\text {quat }}\right), 140.3\left(\mathrm{C}_{\text {quat }}\right), 143.7(\mathrm{CH}), 150.7\left(\mathrm{C}_{\text {quat }}\right), 157.4\left(\mathrm{C}_{\text {quat }}\right), 162.9$ (C $\left.\mathrm{C}_{\text {quat }}\right)$. MS (MALDI-TOF) calcd for $\mathrm{C}_{22} \mathrm{H}_{18} \mathrm{~N}_{2} \mathrm{OS} \mathrm{m} / z: 358.11$; Found: 359.1 ([MH] $\left.]^{+}\right)$IR: $\tilde{v}\left[\mathrm{~cm}^{-1}\right]$ $=2980(\mathrm{w}), 2972(\mathrm{w}), 2918(\mathrm{w}), 2851(\mathrm{w}), 1678(\mathrm{~s}), 1653(\mathrm{w}), 1593(\mathrm{~m}), 1557(\mathrm{~m}), 1500(\mathrm{~m})$, $1489(\mathrm{~m}), 1431(\mathrm{~m}), 1410(\mathrm{~m}), 1375(\mathrm{~m}), 1360(\mathrm{~m}), 1335(\mathrm{w}), 1314(\mathrm{~m}), 1304(\mathrm{~m}), 1255(\mathrm{w})$, $1213(\mathrm{~m}), 1142(\mathrm{~m}), 1096(\mathrm{w}), 1078(\mathrm{~m}), 1024(\mathrm{~m}), 1001(\mathrm{~m}), 957(\mathrm{w}), 926(\mathrm{~m}), 912(\mathrm{w}), 891(\mathrm{w})$, $804(\mathrm{~s}), 797(\mathrm{~s}), 762(\mathrm{~m}), 756(\mathrm{~s}), 729(\mathrm{w}), 689(\mathrm{~s}), 673(\mathrm{~m}), 658(\mathrm{~m}), 619(\mathrm{w})$. Anal calcd for $\mathrm{C}_{22} \mathrm{H}_{18} \mathrm{~N}_{2} \mathrm{OS}$ [358.5]: C 73.71, H 5.06, N 7.82, S 8.95; Found: C 73.63, H 5.09, N 7.86, S 8.98.

\section{Conclusions}

In summary we have elaborated a concise Suzuki coupling Knoevenagel condensation one-pot synthesis of boronic acids/esters, (hetero)aromatic bromo aldehydes and methylene active compounds in the sense of a consecutive three-component process giving rise to the formation products that are merocyanines due to the donor nature of the bromo aldehydes or the Suzuki intermediates in moderate to excellent yield. This synthetic one-pot synthesis is so general that it can be applied as a tool to access huge substance libraries suitable for hit screening and lead finding. As examples, we conducted correlation analyses with consanguineous series by plotting their optical band gap (determined from the intersection of absorption and emission spectra) against the first oxidation potentials (determined by cyclic voltammetry). Indeed, linear correlations could be established for consanguineous of systems with varying the donor part with the same acceptor part. In the sense of a two parameter planar correlation for 24 representatives of four different series a common two parameter planar correlation was found upon plotting the optical band gap energy $E_{0-0}$ against the oxidation potential $E_{1 / 2}$ and the emission maximum $\lambda_{\text {max,em. }}$. Both the synthetic concept warranting rapid access to substance libraries and experimentallybased correlation analyses of consanguineous series, and also of combinations of several series, set the stage for establishing extensive structure-property relationships of chromophores. Further studies are currently underway. 
Supplementary Materials: The following are available online, Supporting Information: Synthetic and analytic details, and ${ }^{1} \mathrm{H}$ and ${ }^{13} \mathrm{C}$ NMR spectra of compounds $\mathbf{8 - 1 2}$, and $\mathbf{1 4}-\mathbf{1 7}$, correlation analysis of the different series.

Author Contributions: The work consists of parts of the Ph. D. thesis of T.M., which was supervised by T.J.J.M., and the research practicum of R.K., which was supervised by T.M. The conceptualization was outlined and accompanied by T.J.J.M. Synthetic studies of compounds 8-12 and 14-17 were performed by T.M. (72\% of the syntheses), R.K. (20\% of the compounds) and experimental support by Francesco König in his B.Sc. thesis ( $8 \%$ of the compounds), which was supervised by T.M. and T.J.J.M. All analytical assignments, electroanalytical and photophysical investigations were performed by T.M., who compiled and interpreted the obtained data. Writing of the original draft was done by T.J.J.M., and review and editing by T.M. and R.K. Project administration and funding acquisition was done by T.J.J.M. and T.M. (scholarship application with Evonik Industries). All authors have read and agreed to the published version of the manuscript.

Funding: The authors thank the Evonik Stiftung (stipend for T.M.), Deutsche Forschungsgemeinschaft (MU 1088/9-1), and the Fonds der Chemischen Industrie for financial support.

Institutional Review Board Statement: Not applicable.

Informed Consent Statement: Not applicable.

Data Availability Statement: All data of this work are included in the manuscript and the Supplementary Materials. UV/VIS and fluorescence spectra as well as cyclic voltammograms can be provided from the authors upon request.

Acknowledgments: The authors thank Francesco König, B. Sc., for experimental assistance in the course of his B.Sc. thesis.

Conflicts of Interest: The authors declare no conflict of interest. The funders had no role in the design of the study, in the collection, analyses, or interpretation of data, in the writing of the manuscript, or in the decision to publish the results.

Sample Availability: Samples of the compounds are not available from the authors.

\section{References}

1. Zhu, J.; Wang, Q.; Wang, M.-X. Multi-Component Reactions in Organic Synthesis; Wiley-VHC: Weinheim, Germany, 2015. [CrossRef]

2. Müller, T.J.J. Science of Synthesis Series: Multicomponent Reactions 1 - General Discussion and Reactions Involving a Carbonyl Compound as Electrophilic Component; Müller, T.J.J., Ed.; Georg Thieme: Stuttgart, Germany, 2014; pp. 5-27. [CrossRef]

3. D'Souza, D.M.; Müller, T.J.J. Multi-Component Syntheses of Heterocycles by Transition Metal Catalysis. Chem. Soc. Rev. 2007, 36, 1095-1108. [CrossRef]

4. Dömling, A.; Ugi, I. Multicomponent Reactions with Isocyanides. Angew. Chem. Int. Ed. 2000, 39, 3169-3210. [CrossRef]

5. Levi, L.; Müller, T.J.J. Multicomponent syntheses of functional chromophores. Chem. Soc. Rev. 2016, 45, 2825-2846. [CrossRef]

6. Müller, T.J.J.; Bunz, U.H.F. Functional Organic Materials; Wiley-VHC: Weinheim, Germany, 2007. [CrossRef]

7. Levi, L.; Müller, T.J.J. Multicomponent Syntheses of Fluorophores Initiated by Metal Catalysis. Eur. J. Org. Chem. 2016, 2016, 2907-2918. [CrossRef]

8. Shindy, H.A. Fundamentals in the chemistry of cyanine dyes: A review. Dyes Pigm. 2017, 145, 505-513. [CrossRef]

9. Panigrahi, M.; Dash, S.; Patel, S.; Mishra, B.K. Syntheses of Cyanines: A Review. Tetrahedron 2012, 68, 781-805. [CrossRef]

10. Kulinich, A.V.; Ishchenko, A.A. Merocyanine dyes: Synthesis, structure, properties and applications. Russ. Chem. Rev. 2009, 78, 141-164. [CrossRef]

11. Shirinian, V.Z.; Shimkim, A.A. Merocyanines: Synthesis and Application. Top. Heterocycl. Chem. 2008, 14, 75-105. [CrossRef]

12. Mishra, A.; Behera, R.K.; Behera, P.K.; Mishra, B.K.; Behera, G.B. Cyanines during the 1990s: A Review. Chem. Rev. 2000, 100, 1973-2011. [CrossRef]

13. Hamer, F.M. The Cyanine Dyes and Related Compounds. Available online: https://onlinelibrary.wiley.com/doi/book/10.1002/ 9780470186794 (accessed on 16 July 2021).

14. Kim, T.-D.; Lee, K.-S. D- $\pi-A$ Conjugated Molecules for Optoelectronic Applications. Macromol. Rapid Commun. 2015, 36, 943-958. [CrossRef] [PubMed]

15. Castet, F.; Rodriguez, V.; Pozzo, J.-L.; Ducasse, L.; Plaquet, A.; Champagne, B. Design and Characterization of Molecular Nonlinear Optical Switches. Acc. Chem. Res. 2013, 46, 2656-2665. [CrossRef] [PubMed]

16. Marder, S.R. Organic nonlinear optical materials: Where we have been and where we are going. Chem. Commun. 2006, 131-134. [CrossRef] 
17. Würthner, F.; Wortmann, R.; Meerholz, K. Chromophore design for photorefractive organic materials. ChemPhysChem 2002, 3, 17-31. [CrossRef]

18. Gsänger, M.; Bialas, D.; Huang, L.; Stolte, M.; Würthner, F. Organic Semiconductors based on Dyes and Color Pigments. Adv. Mater. 2016, 28, 3615-3645. [CrossRef] [PubMed]

19. Leo, K. Elementary Processes in Organic Photovoltaics; Springer: Cham, Switzerland, 2017; pp. 193-214. [CrossRef]

20. Würthner, F. Dipole-Dipole Interaction Driven Self-Assembly of Merocyanine Dyes: From Dimers to Nanoscale Objects and Supramolecular Materials. Acc. Chem. Res. 2016, 49, 868-876. [CrossRef] [PubMed]

21. Heyne, B. Self-assembly of organic dyes in supramolecular aggregates. Photochem. Photobiol. Sci. 2016, 15, 1103-1114. [CrossRef]

22. Kovtun, Y.P.; Prostota, Y.O.; Shandura, M.P.; Poronik, Y.M.; Tolmachev, A.I. Metallochromic merocyanines of 8-hydroxyquinoline series. II. Dyes with end nuclei of low basicity. Dyes Pigm. 2004, 60, 215-221. [CrossRef]

23. Kovtun, Y.P.; Prostota, Y.O.; Tolmachev, A.I. Metallochromic merocyanines of 8-hydroxyquinoline series. Dyes Pigm. 2003, 58, 83-91. [CrossRef]

24. Yagi, S.; Maeda, K.; Nakazumi, H.J. Photochromic properties of cationic merocyanine dyes. Thermal stability of the spiropyran form produced by irradiation with visible light. Mater. Chem. 1999, 9, 2991-2997. [CrossRef]

25. Elsner, A.-L.; Biesen, L.; Müller, T.J.J. Pseudo-Five-component Synthesis of Indolone-3-aminopropenylidene Merocyanine Dimers and Their Attenuated Aggregation-Induced Emission. ARKIVOC 2021, 53-66. [CrossRef]

26. D'Souza, D.M.; Muschelknautz, C.; Rominger, F.; Müller, T.J.J. Unusual Solid-State Luminescent Push-Pull Indolones: A general One-Pot Three-component Approach. Org. Lett. 2010, 12, 3364-3367. [CrossRef]

27. Denißen, M.; Hannen, R.; Itskalov, D.; Biesen, L.; Nirmalananthan-Budau, N.; Hoffmann, K.; Reiss, G.J.; Resch-Genger, U.; Müller, T.J.J. One-pot Synthesis of a White-light Emissive Bichromophore Operated by Aggregation-induced Dual Emission (AIDE) and Partial Energy Transfer. Chem. Commun. 2020, 56, 7407-7410. [CrossRef] [PubMed]

28. Pasch, P.; Papadopoulos, J.; Goralczyk, A.; Hofer, M.L.; Tabatabai, M.; Müller, T.J.J.; Hartmann, L. Highly fluorescent merocyanine and cyanine PMMA copolymers. Macromol. Rapid Commun. 2018, 39, 1800277. [CrossRef] [PubMed]

29. Muschelknautz, C.; Frank, W.; Müller, T.J.J. Rapid Access to Unusual Solid-State Luminescent Merocyanines by a Novel One-Pot Three-component Synthesis. Org. Lett. 2011, 13, 2556-2559. [CrossRef]

30. Papadopoulos, J.; Merkens, K.; Müller, T.J.J. Three-Component Synthesis and Photophysical Properties of Novel Coumarin-based Merocyanines. Chem. Eur. J. 2018, 24, 974-983. [CrossRef] [PubMed]

31. Meyer, T.; Müller, T.J.J. Consecutive Three-Component Synthesis of Donor-Substituted Merocyanines by One-pot SuzukiKnoevenagel Condensation (SuKnoCon) Sequence. Org. Mater. 2020, 2, 64-70. [CrossRef]

32. Park, S.Y.; Park, G.E.; Choi, S.; Lee, J.H.; Lee, D.H.; Cho, M.J.; Choi, D.H. Effect of acceptor strength in new acceptor-donoracceptor-type molecules on their miscibility with donor polymers for bulk-heterojunction fullerene-free solar cells. Dyes Pigm. 2017, 146, 226-233. [CrossRef]

33. Hedström, S.; Wang, E.; Persson, P. Defining donor and acceptor strength in conjugated copolymers. Mol. Phys. 2017, 115, 485-496. [CrossRef]

34. Zanello, P. Electrochemical and X-ray Structural Aspects of Transition Metal Complexes Containing Redox-Active Ferrocene Ligands in Ferrocenes; Togni, A., Hayashi, T., Eds.; Wiley VHC: Weinheim, Germany, 1995; p. 317. [CrossRef] 